Michelle MAtOs ${ }^{1}$

LeDA FERRAZ ${ }^{2}$

PAtríCIA de FÁtima LOPES ${ }^{3}$

CONSUELO LOZOYA ${ }^{3}$

JOFFRE AMIM JUNIOR ${ }^{4}$

JORGE REZENDE-FILHO

ANTONIO BRAGA ${ }^{4,5}$

\title{
Neoplasia trofoblástica gestacional após
} normalização espontânea da gonadotrofina coriônica humana em paciente com mola hidatiforme parcial

\section{Gestational trophoblastic neoplasia after spontaneous normalization of buman chorionic gonadotropin in patient with partial bydatidiform mole}

Relato de Caso

Doença trofoblástica gestacional/diagnóstico Doença trofoblástica gestacional/quimioterapia

Mola hidatiforme

Gonadotropina coriônica

Keywords

Gestational trophoblastic disease/diagnosis Gestational trophoblastic disease/drug therapy Hydatidiform mole Chorionic gonadotropin

Correspondêncio

Antonio Brago

Centro de Doencas Trofoblásticas do Rio de Janeiro, Maternidade Escola da Universidade Federal do Rio de Janeiro Rua das Laranjeiras, 180 - Laranjeiras

CEP: 22240-004

Rio de Janeiro (RJ), Brasil

Recebido

27/02/2015

Aceito com modificacōes

$01 / 06 / 2015$

\section{Resumo}

Neste relato, é apresentado um caso de neoplasia trofoblástica gestacional após normalização espontânea de gonadotrofina coriônica humana em paciente com mola hidatiforme parcial. Trata-se da segunda ocorrência publicada desse evento e a primeira em que há comprovação imuno-histoquímica. No bojo dessa apresentação, ademais de mostrar o tratamento para essa intercorrência da gravidez, discute-se a possibilidade de redução da duração do seguimento pós-molar, assim como estratégias para o precoce reconhecimento da neoplasia trofoblástica gestacional após a remissão espontânea da gravidez molar.

\section{Abstract}

We report here a case of gestational trophoblastic neoplasia after spontaneous normalization of human chorionic gonadotropin in a patient with a partial hydatidiform mole. This is the second occurrence of this event to be reported and the first one with proven immunohistochemical evidence. Besides showing the treatment for this pregnancy complication, this case report discusses the possibility of reducing the duration of post-molar follow-up, as well as strategies for early recognition of gestational trophoblastic neoplasia after spontaneous remission of molar pregnancy.
Centro de Doença Trofoblástica Gestacional, Maternidade Escola, Universidade Federal do Rio de Janeiro - UFRJ - Rio de Janeiro (RJ), Brasil. 'Programa de Mestrado Profissional em Saúde Materno-Infantil, Universidade Federal Fluminense - UFF - Niterói (RJ), Brasil.

2Programa de Doutorado em Ciências Médicas, Universidade Federal Fluminense - UFF - Niterói (RJ), Brasil. ${ }^{3}$ Departamento de Patologia, Faculdade de Medicina, Universidade Federal Fluminense - UFF - Niterói (RI), Brasil.

${ }^{4}$ Departamento de Ginecologia e Obstetrícia, Faculdade de Medicina, Universidade Federal do Rio de Janeiro - UFRj; Programa de Mestrado Profissional em Saúde Perinatal, Maternidade Escola, Universidade Federal do Rio de Janeiro - UFRJ - Rio de Janeiro (RJ), Brasil. ${ }^{5}$ Centro de Doença Trofoblástica Gestacional, Maternidade Escola, Universidade Federal do Rio de Janeiro - UFRJ - Rio de Janeiro (RJ): Hospital Universitário Antonio Pedro, Universidade Federal Fluminense - UFF - Rio de Janeiro (RI); Departamento de Ginecologia, Brasil. Conflito de interesses: não há. 


\section{Introdução}

A doença trofoblástica gestacional (DTG) é uma anomalia da gestação que acomete cerca de 1:200 grávidas brasileiras ${ }^{1,2}$, apresentando formas clínicas benignas, representada pela mola hidatiforme completa (MHC) e parcial (MHP) e um espectro maligno chamado neoplasia trofoblástica gestacional (NTG) ${ }^{3-5}$.

Certamente, o grande desafio do acompanhamento dessas pacientes é garantir a adesão à vigilância hormonal de gonadotrofina coriônica humana (hCG), cuja monitoração periódica permite o diagnóstico da NTG e o início precoce da quimioterapia apropriada ${ }^{3,4}$. Isso pode ser especialmente difícil nos países subdesenvolvidos ou naqueles que imperam grandes distâncias territoriais ${ }^{2,6,7}$, determinando que um grande número de mulheres abandonem o acompanhamento $0^{2,6,8-10}$.

A fim de minorar essa situação, tem-se aventado a redução da duração do seguimento pós-molar, que hoje implica em dosagens semanais de hCG até sua normalização por três semanas consecutivas, quando passa a ser feito mensalmente por, ao menos, seis meses ${ }^{3,4}$, chegando até a um a dois anos em alguns Serviços ${ }^{11-13}$.

Essa proposta liberaria as pacientes do seguimento pós-molar estendido após a normalização de hCG, a partir da qual o risco de transformação maligna seria negligível ${ }^{6,10,11,13-21}$. Esse cenário de redução da duração do seguimento pós-molar tem sido mais enfatizado entre pacientes com MHP que alcançaram a normalização do $\mathrm{hCG}^{6,10,11,15-21}$, para o qual a literatura apresenta apenas um único relato de caso de NTG subsequente ${ }^{13}$.

Todavia, Bagshawe et al. ${ }^{14}$ apresentaram, pela primeira vez, o relato de pacientes com NTG após normalização dos níveis de hCG, recomendando um seguimento pós-molar

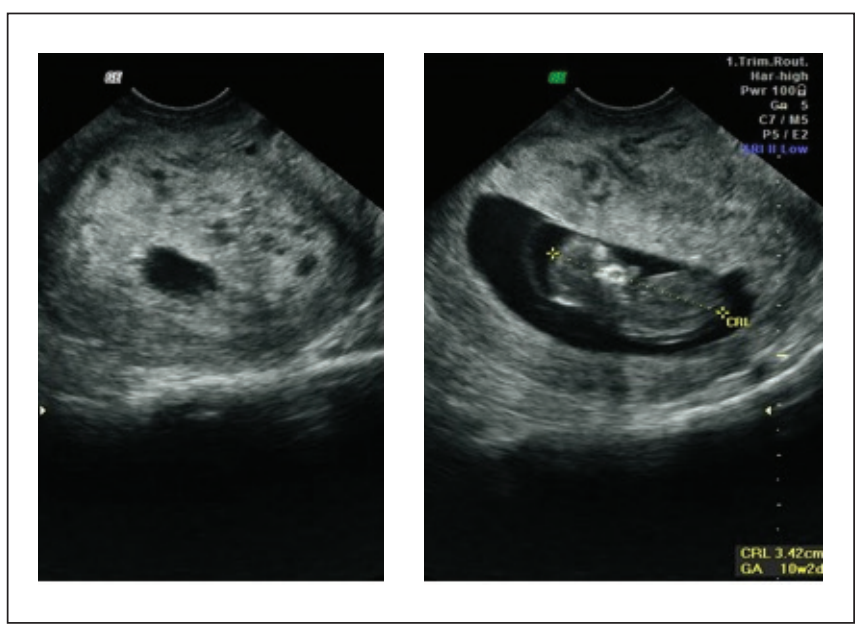

Figura 1. Ultrassonografia mostrando imagem de mola hidatiforme parcial. Nota-se a presença de embrião em um saco gestacional com decídua de aspecto de "queijo suíço", cujas áreas anecogênicas representam as vesículas hidatiformes prolongado, ainda vigente. Esses casos, conquanto pouco frequentes, são reportados em muitos dos trabalhos que investigam esse tema, trazendo em seu bojo a dúvida sobre a real segurança da interrupção precoce do seguimento pós-molar.

É objetivo deste relato apresentar a história de uma paciente que cursou com normalização espontânea de hCG em seu seguimento pós-molar e que desenvolveu NTG subsequente. Esse é o segundo caso dessa ocorrência em pacientes com MHP e o primeiro com confirmação imuno-histoquímica ${ }^{13}$, segundo ampla revisão entre artigos ocidentais sobre o tema $a^{10,11,13,15-21}$.

\section{Descrição do caso}

Este relato de caso foi obtido de estudo aprovado pelo Comitê de Ética em Pesquisa da Maternidade Escola da Universidade Federal do Rio de Janeiro, sob o protocolo 572.887.

Paciente com 25 anos, nulípara, percebe amenorreia em maio de 2012. Apenas em agosto de 2012, após vultosa hemorragia, procura atendimento médico, sendo submetida à ultrassonografia transvaginal que exarou hipótese de MHP, como mostra a Figura 1. A paciente foi encaminhada ao Centro de Referência em DTG do Rio de Janeiro, sendo submetida ao esvaziamento uterino com saída de abundante quantidade de material, sugestivo de mola hidatiforme parcial, pela presença de partes fetais evidenciadas à macroscopia. Após análise histopatológica e imuno-histoquímica com estudo do $\mathrm{p} 57^{\mathrm{kip} 2}$, foi confirmando o diagnóstico de MHP, como pode ser visto nas Figuras 2 e 3.

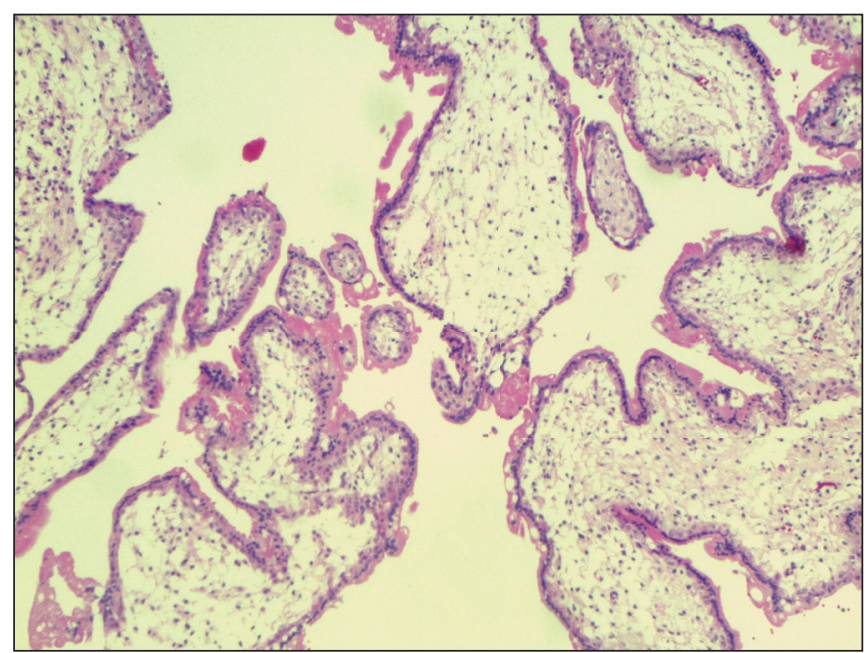

Figura 2. Microscopia da mola hidatiforme parcial. São visualizadas vilosidades em dupla população, ora vilosidades pequenas, ora com contornos irregulares, com inclusões e invaginações do trofoblasto, apresentando edema estromal, com hiperplasia do citotrofoblasto e sinciciotrofoblasto com discretas a moderadas atipias. HE 10x 
Iniciado o seguimento pós-molar, salienta-se que todas as dosagens de hCG foram feitas no mesmo Serviço e com o mesmo conjunto de insumos para diagnóstico (Diagnostic Products Corporation Immulite ${ }^{\circledR}$ da Siemens, sendo considerado valor de referência para resultados normais a dosagem de hCG inferior a $5 \mathrm{mUI} / \mathrm{mL}$ ). Sua dosagem inicial de hCG foi de $19.800 \mathrm{mUI} / \mathrm{mL}$ e decresceu semanalmente, consoante a curva de eliminação normal de $\mathrm{hCG}^{22}$, alcançando a remissão espontânea em outubro de 2012, após 7 semanas do esvaziamento uterino. A paciente manteve níveis normais de hCG por 6 meses, quando recebeu alta do seguimento pós-molar em 20 de março de 2013.

Em maio de 2013, 7 meses após a normalização de hCG e 2 meses após a alta do seguimento pós-molar, a paciente retornou ao Centro de Referência em DTG do Rio de Janeiro queixando-se de amenorreia. Ela estava preocupada, pois referiu não ter tido relações sexuais desde o esvaziamento uterino. Foram realizadas dosagens de hCG, nos dias 2, 8, 15 e 20 de maio de 2013, exibindo os seguintes resultados, respectivamente: $120,290,380$ e $987 \mathrm{mUI} / \mathrm{mL}$, sendo diagnosticada NTG.

A paciente foi submetida a uma criteriosa avaliação clínica-laboratorial-radiológica, sendo sua NTG estadiada como I:4 (idade < 40 anos=0; antecedente obstétrico de mola hidatiforme $=0$; intervalo em meses entre a terminação da gravidez e o diagnóstico da NTG $\geq 12$ meses=4; nível de hCG pré-tratamento $<10^{3} \mathrm{mUI} / \mathrm{mL}=0$; com doença limitada ao útero, não podendo a lesão miometrial ser mensurada $=0$ ). Salientamos que o exame pélvico e a radiografia de tórax estavam normais. Ainda que não seja

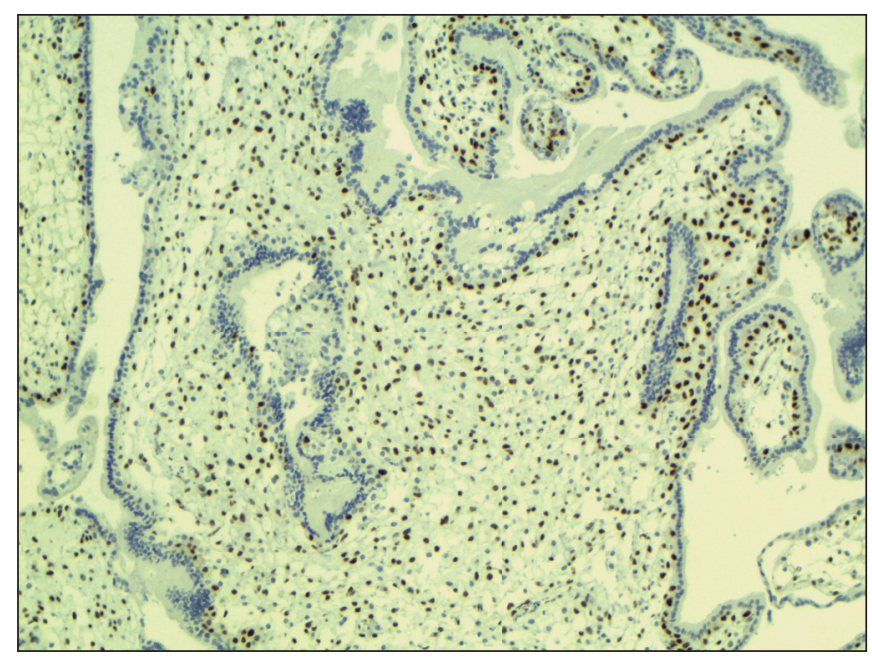

Figura 3. Imuno-histoquímica da mola hidatiforme parcial, pelo anticorpo anti-p57kip2. A expressão do p57kip2 é a caracterização gênica de alelo paterno de impressão materna, ausente nos casos de mola hidatiforme completa (que é um partenogenoma), mas presente fortemente no citotrofoblasto, estroma das vilosidades coriônicas, como aqui exibido neste caso de mola hidatiforme parcial (que é uma triploidia diândrica). IHQ p57kip2 10x. feita rotineiramente em pacientes no estadio I, devido à história clínica excepcional, foi realizada tomografia computadorizada de tórax, ressonância nuclear magnética de crânio e abdome, que não mostravam sinais de metástase.

O tratamento antineoplásico foi imediatamente instituído, utilizando-se o regime Methotrexate (MTX) $1 \mathrm{mg} / \mathrm{kg}$ de peso via intramuscular nos dias $1,3,5$ e 7; alternado com o ácido folínico (FC) $15 \mathrm{mg}$ via oral nos dias 2, 4, 6 e 8, ingeridos 24 h após a injeção do quimioterápico (MTX/FC), conforme pode ser observado na Tabela 1. Esse tratamento foi repetido a cada 7-14 dias. Imediatamente antes de iniciar-se o próximo ciclo de quimioterapia, era dosado hCG e avaliada a toxicidade do tratamento, mediante análise do hemograma completo com plaquetometria, ureia, creatinina, ácido úrico, aspartato aminotransferase e alanina aminotransferase. A paciente apresentou após o $2^{\circ}$ ciclo de MTX/FC anemia grau 1 , síndrome do olho seco e mucosite oral grau II, consoante os critérios de toxicidade elaborados pelo National Institute of Health e National Cancer Institute ${ }^{23}$ e tratados ambulatorialmente. Foram necessárias sete semanas de tratamento e quatro ciclos de MTX/FC, para que a paciente alcançasse a remissão, após a qual foi submetida ainda a mais três ciclos de quimioterapia de consolidação com o mesmo regime antineoplásico.

A paciente encontra-se com mais de 12 meses em remissão sustentada, sendo orientada para a necessidade

Tabela 1. Resumo do tratamento de neoplasia trofoblástica gestacional após a normalizacãa espontânea da gonadotrofina coriônica humana no seguimento de paciente com mola hidatiforme parcial

\begin{tabular}{|c|c|c|}
\hline Data & $\begin{array}{c}\text { Gonadotrofina coriônica } \\
\text { humana* }\end{array}$ & Conduta \\
\hline $20-27 / 05 / 2013$ & - & MTX/FC** \\
\hline $03 / 06 / 2013$ & 320 & - \\
\hline $3-10 / 06 / 2013$ & - & MTX/FC** \\
\hline $17 / 06 / 2013$ & 110 & - \\
\hline $17-24 / 06 / 2013$ & - & MTX/FC** \\
\hline $01 / 07 / 2013$ & 26 & - \\
\hline $1-8 / 07 / 2013$ & - & MTX/FC** \\
\hline $15 / 07 / 2013$ & 2 & - \\
\hline $15-22 / 07 / 2013$ & - & $\mathrm{MTX} / \mathrm{FC}^{\star \star \star \star}$ \\
\hline $29 / 07 / 2013$ & $<0,1$ & - \\
\hline $29 / 07-5 / 08 / 2013$ & - & MTX/FC \\
\hline $12 / 08 / 2013$ & $<0,1$ & - \\
\hline $12-19 / 08 / 2013$ & - & MTX/FC \\
\hline $26 / 08 / 2013^{* * * *}$ & $<0,1$ & - \\
\hline $14 / 01 / 2015$ & $<0,1$ & Alta do seguimento ${ }^{\star \star \star \star \star}$ \\
\hline
\end{tabular}

*Dosagem de gonadotrofina coriônica humana em $\mathrm{mUI} / \mathrm{mL}$; ${ }^{*}$ *MTX/FC: Methotrexate com resgate do ácido folínico; ${ }^{* * *}$ quimioterapia de consolidação; ****foram realizadas dosagens mensais de gonadotrofina coriônica humana entre os meses de agosto de 2013 e agosto de 2014 , com resultados $<0,1 \mathrm{mUl} / \mathrm{mL}$; ***** alta do seguimento = monitoração da gonadotrofina coriônica humana passa a ser feita anualmente, ao menos por cinco anos, idealmente pelo resto da vida. 
de monitoração bioquímica, ao menos durante 5 anos, idealmente por toda a vida.

\section{Discussão}

Inúmeros estudos têm sugerindo que a duração do seguimento pós-molar deve ser revista ${ }^{6,8,9,11,24}$, pela ínfima chance de 0,27\% da ocorrência de NTG após a normalização espontânea de hCG ${ }^{10,11,13,15-21}$. Quando se consideram apenas os casos de MHP, essa prevalência é ainda menor, não justificando uma vigilância hormonal estendida ${ }^{10,11,13,15-21}$. Houve apenas um caso de NTG em paciente após a remissão de MHP, oriundo do Senegal. Dos estudos que investigaram esse tema, esse é o segundo que apresenta essa possibilidade $e^{10,11,13,15-21}$, sendo que o primeiro deles não menciona a realização de técnicas de apoio histopatológico para essa confirmação diagnóstica ${ }^{13}$. Sabe-se que a MHP pode facilmente ser confundida com MHC de idade gestacional precoce, sendo necessária nesses casos a complementação da histopatologia pela imuno-histoquímica ou citogenética ${ }^{3,4}$, como realizado por nós.

Não sendo exequível o diagnóstico da NTG, após a remissão espontânea, mediante vigilância hormonal prolongada, dada a baixa adesão ao acompanhamento hormonal $^{2,6,8,10,15,16,19}$, seria fundamental que outras estratégias pudessem ser consideradas para o precoce reconhecimento desses casos de NTG.

Dados da literatura mostram que as pacientes com NTG após a normalização de hCG somente foram diagnosticadas porque retornaram ao Centro de Referência com alguma queixa relacionada à gravidez (amenorreia, náusea) ou ao surgimento de manifestações clínicas da neoplasia ou de suas metástases (hemorragia uterina, hemoptise, dispneia). Na casuística francesa, 5 de $6(83 \%)$ pacientes apresentaram alguma sintomatologia que antecipou o diagnóstico da NTG: 3/5 (60\%) tiveram amenorreia (como a da nossa paciente) e 2 de 5 (40\%), hemorragia uterina ${ }^{21}$, reforçando a necessidade de orientar a paciente por ocasião da alta do seguimento para retornar ao Centro de Referência no surgimento de qualquer uma das sintomatologias apresentadas, não apenas para descartar gravidez, como também NTG após a normalização de hCG.

Uma vez diagnosticada a NTG após a remissão, o estadiamento, a análise dos fatores prognósticos e o tratamento não difere do que é empregado para as pacientes com NTG pós-molar. Sabe-se que pacientes com doença não precocemente detectada (como acontece com as pacientes com NTG após a remissão da gravidez molar) ${ }^{21}$ têm seu prognóstico empiorado, o que não aconteceu com nossa paciente. Considerando-se os casos reportados pelos estudos da Hungria, França e Senegal, houve maior prevalência de NTG de alto risco $(50 \%)^{10,13,21}$, notadamente pelo grande intervalo entre o término da gravidez molar e o início da quimioterapia.

Nos casos em que há indicação para cirurgia, parece-nos de bom alvitre fazer minucioso estudo histopatológico e imuno-histoquímico com a avaliação do lactogênio placentário humano a fim de diagnosticar o tumor trofoblástico do sítio placentário, forma rara de NTG, presente em $16 \%$ das pacientes da casuística francesa ${ }^{21}$.

Nosso relato apresenta algumas limitações que devem ser salientadas. A primeira dela diz respeito ao conjunto de insumos para diagnóstico comercial usado para a dosagem de hCG, que é voltado apenas para diagnosticar a gravidez, não desenvolvido para dosar todas suas frações ${ }^{21,25}$. A segunda é a ausência de testes genéticos para afastar a possibilidade de que a NTG de nossa paciente tenha se originado de outra gravidez ${ }^{21}$. Todavia, essas limitações são comuns a todos os estudos anteriores desse tema ${ }^{6,10,11,13-21}$.

Não existe, no momento, ensaio clínico ou melhor evidência que assegure o tempo ideal para a duração do seguimento pós-molar. Sabe-se que o risco de NTG após a normalização de hCG é ínfimo e, quando ela ocorre, dificilmente a vigilância hormonal habitual de seis meses será capaz de diagnosticá-la. Assim, no Centro de Doenças Trofoblásticas do Rio de Janeiro, temos acompanhado as pacientes com MHC por três a seis meses (a depender do tempo para a normalização de hCG); já aquelas com MHP são liberadas do seguimento pós-molar por ocasião da terceira dosagem de hCG menor que $5 \mathrm{mUI} / \mathrm{mL}$.

$\mathrm{Na}$ alta do seguimento pós-molar, as pacientes devem ser orientadas a retornar ao Centro de Referência diante de qualquer anormalidade clínica, notadamente aquelas relacionadas com presença de gravidez, lesão genital ou metástases a fim de realizar-se dosagem de hCG, diagnosticar gravidez ou afastar a transformação maligna. Essa parece ser a estratégia mais efetiva para o diagnóstico precoce de NTG após a remissão em nossas pacientes, uma vez que a vigilância hormonal estendida é pouco exequível para nossa população brasileira.

\section{Agradecimentos}

À Dra. Valéria Moraes, do Hospital Universitário Antonio Pedro da Universidade Federal Fluminense, responsável pela cessão das imagens de ultrassonografia (Figura 1). 
1. Belfort $P$, Braga A. [The changing clinical presentation of molar pregnancy]. Rev Bras Ginecol Obstet. 2004;26(6):483-8. Portuguese.

2. Braga A, Uberti EM, Fajardo MC, Viggiano M, Sun SY, Grillo $B M$, et al. Epidemiological report on the treatment of patients with gestational trophoblastic disease in 10 Brazilian referral centers: results after 12 years since International FIGO 2000 consensus. J Reprod Med. 2014;59(5-6):241-7.

3. Seckl M, Sebire NJ, Fisher RA, Golfier F, Massuger L, Sessa C, et al. Gestational trophoblastic disease: ESMO Clinical Practice Guidelines for diagnosis, treatment and follow-up. Ann Oncol. 2013;24 Suppl 6:vi39-50.

4. Berkowitz RS, Goldstein DP. Current advances in the management of gestational trophoblastic disease. Gynecol Oncol. 2013;128(1):3-5.

5. Biscaro A, Braga A, Berkowitz RS. Diagnosis, classification and treatment of gestational trophoblastic neoplasia. Rev Bras Ginecol Obstet. 2015;37(1):42-51

6. Feltmate CM, Batorfi J, Fulop V, Goldstein DP, Doszpod J, Berkowitz RS. Human chorionic gonadotropin follow-up in patients with molar pregnancy: a time for reevaluation. Obstet Gynecol. 2003; 101 (4):732-6.

7. Maestá I, Braga A. [Challenges of the treatment of patients with gestational trophoblastic disease]. Rev Bras Ginecol Obstet. 2012;34(4):143-6. Portuguese.

8. Massad LS, Abu-Rustum NR, Lee SS, Renta V. Poor compliance with postmolar surveillance and treatment protocols by indigent women. Obstet Gynecol. 2000;96(6):940-4.

9. Allen JE, King MR, Farrar DF, Miller DS, Schorge JO. Postmolar surveillance at a trophoblastic disease center that serves indigent women. Am J Obstet Gynecol. 2003; 188(5):1151-3.

10. Batorfi J, Vegh G, Szepesi J, Szigetvari I, Doszpod J, Fulop V. How long should patients be followed after molar pregnancy? Analysis of serum hCG follow-up data. Eur J Obstet Gynecol Reprod Biol. 2004; $112(1): 95-7$.

11. Sebire NJ, Foskett M, Short D, Savage P, Stewart W, Thomson $M$, et al. Shortened duration of human chorionic gonadotrophin surveillance following complete or partial hydatidiform mole: evidence for revised protocol of a UK regional trophoblastic disease unit. BJOG. 2007;114(6):760-2.

12. Institut National du Cancer. Maladies trophoblastiques gestationnelles, collection recommandations et référentiels, ouvrage collectif édité par I'INCa. Boulogne-Billancourt: Institut National du Cancer; 2010.
13. Gueye M, Kane-Gueye SM, Ndiaye-Gueye MD, Mbaye M, Diouf AA, Niang MM, et al. Gestational trophoblastic neoplasia after achieving a nondetectable serum human chorionic gonadotrophin level. BJOG. 2014;121(11):1415-9.

14. Bagshawe KD, Dent J, Webb J. Hydatidiform mole in England and Wales 1973-83. Lancet. 1986;2(8508):673-7.

15. Wolfberg AJ, Feltmate C, Goldstein DP, Berkowitz RS, Lieberman E. Low risk of relapse after achieving undetectable HCG levels in women with complete molar pregnancy. Obstet Gynecol. 2004;104(3):551-4.

16. Lavie I, Rao GG, Castrillon DH, Miller DS, Schorge JO. Duration of human chorionic gonadotropin surveillance for partial hydatidiform moles. Am J Obstet Gynecol. 2005;192(5):1362-4.

17. Wolfberg AJ, Growdon WB, Feltmate CM, Goldstein DP, Genest $D R$, Chinchilla $M E$, et al. Low risk of relapse after achieving undetectable HCG levels in women with partial molar pregnancy. Obstet Gynecol. 2006;108(2):393-6.

18. Kerkmeijer L, Wielsma S, Bekkers R, Pyman J, Tan J, Quinn M. Guidelines following hydatidiform mole: a reappraisal. Aust N Z J Obstet Gynaecol. 2006;46(2): $112-8$.

19. Wiesma S, Kerkmeijer L, Bekkers R, Pyman J, Tan J, Quinn M. Persistent trophoblast disease following partial molar pregnancy. Aust N Z J Obstet Gynaecol. 2006;46(2):1 19-23.

20. Kerkmeijer LG, Wielsma S, Massuger LF, Sweep FC, Thomas CM. Recurrent gestational trophoblastic disease after hCG normalization following hydatidiform mole in The Netherlands. Gynecol Oncol. 2007; 106(1): 142-6.

21. Schmitt C, Doret M, Massardier J, Hajri T, Schott AM, Raudrant $D$, et al. Risk of gestational trophoblastic neoplasia after hCG normalisation according to hydatidiform mole type. Gynecol Oncol. 2013;130(1):86-9.

22. Delmanto LRMG, Maestá I, Braga Neto AR, Michelin OC, Passos JRS, Gaiotto FR, et al. [Are curves of human chorionic gonadotropin useful in the early diagnosis of post-molar trophoblastic neoplasia?] Rev Bras Ginecol Obstet. 2007;29(10):506-10. Portuguese.

23. National Institutes of Health. National Cancer Institute [Internet]. Common Terminology Criteria for Adverse Events (CTCAE). Version 4.0. 2009 [cited 2015 Feb 18]. Available from: <http://evs.nci.nih.gov/ ftpl/CTCAE/CTCAE_4.03_2010-06-14_QuickReference_5x7.pdf>

24. Pisal N, Tidy J, Hancock B. Gestational trophoblastic disease: is intensive follow up essential in all women? BJOG. 2004; 111 (12):1449-51.

25. Cole LA, Kohorn El. The need for an hCG assay that appropriately detects trophoblastic disease and other hCG-producing cancers. J Reprod Med. 2006;51(10):793-811. 